\title{
Die Konstanzer Life-Studie
}

\section{B. Renner; G. Sproesser; V. Klusmann; H. Schupp}

Arbeitsgruppe Psychologische Diagnostik und Gesundheitspsychologie, Universität Konstanz,

Die Konstanzer Life-Studie des Verbundforschungsprojekts EATMOTIVE erforscht das normale Essverhalten und sucht Antworten auf die Frage „Warum essen Menschen das, was sie essen?"“. Außerdem soll die Studie Aufschluss darüber geben, wie sich die Ernährung langfristig auf Gesundheit und Wohlbefinden auswirkt.

Zunächst stellt sich die Frage, warum wir uns überhaupt für das normale Essverhalten interessieren sollten. Bisher fokussieren Forschung und Medien nahezu ausschließlich auf das pathologische Essverhalten sowie Übergewicht und seine Folgen. Unter diesen Stichworten finden sich in wissenschaftlichen Datenbanken wie dem Web of Science über 200000 Arbeiten. Dem stehen weniger als 200 Arbeiten zum normalen Essverhalten gegenüber.

Dabei ist das normale Essverhalten ein zentrales, basales Motivationssystem der Menschen, unabhängig davon in welcher Kultur sie aufwachsen. Wir alle starten unsere Nahrungsaufnahme mit genau einem Lebensmittel, aber über die Lebensspanne findet eine atemberaubende Ausdifferenzierung statt. Heutzutage können wir in modernen, westlichen Supermärkten zwischen bis zu 60000 Produkten auswählen.

Allein dies macht das normale Essverhalten zu einem spannenden Forschungsgegenstand. In der Konstanzer Life-Studie stellen wir daher nicht den Aspekt der Störung und Krankheit - wie bei Essstörungen (z.B. Anorexia nervosa, Bulimie) oder Adipositas - in den Mittelpunkt, sondern betrachten die „normale“ "Vielfalt des Essverhaltens. So wollen wir anhand von „Erfolgsmodellen“ adaptive Ernährungsmuster identifizieren. Die bessere Kenntnis der zugrundeliegenden Anreiz- bzw. Aversionsfaktoren des normalen Ernährungsverhaltens soll schließlich in zielgruppenspezifische Maßnahmen im Rahmen von Gesundheitsförderungsprogrammen (z.B. von Krankenkassen, Kommunen, Betrieben) überführt werden.

Wir gehen davon aus, dass das normale Essverhalten weit mehr als ,gesundes“ Essen ist und vielfältige Funktionen hat: Kaviar für das Image; Salat, Apfel und Kamillentee für die Gesundheit; Fleisch für den sozialen Status oder auch Schokolade, um die Nerven zu beruhigen. So lernen wir bereits sehr früh, dass mit Essen Wertschätzung ausgedrückt werden kann, dass es wohltuend sein kann oder dass es bestimmte gesellschaftliche Ereignisse kennzeichnet (z.B. der klassische Geburtstagskuchen). Die Frage, warum wir essen, was wir essen, führt vermutlich zu einer komplexen Antwort, der wir in der Konstanzer Life-Studie nachgehen.

Welche Motive der Wahl von Nahrungsmitteln zugrunde liegen, unterscheidet sich von Person zu Person. Daher wird in einem ersten Schritt geklärt, welche Motive für die Ernährung wichtig sind. In einem zweiten Schritt wird untersucht, ob bestimmte Motive eine gesunde und genussvolle Ernährung fördern und wie sich diese wiederum auf die Gesundheit und das Wohlbefinden auswirkt. Besonders wichtig ist es, diese Zusammenhänge in ihrer zeitlichen Entwicklung abzubilden. Daher umfasst die Life-Studie drei Erhebungen über ein Jahr.

\section{Die Konstanzer Life- Studie: Machen Sie mit!}

Erfahren Sie oder Ihre Patienten mehr über ihre Ernährung und Fitness.

Der Check-up dauert etwa zwei Stunden und findet im Bürgersaal der Stadt Konstanz im Stadtzentrum statt.

Weitere Informationen und Anmeldung unter: www.uni-konstanz.de/life-studie

Schreiben Sie uns eine E-Mail: life-studie@uni-konstanz.de oder rufen Sie uns an: 07531/88 5511

Teilnehmen können Sie wieder ab dem 24. September 2012.

\section{Die zentralen Untersuchungsbausteine}

Ablauf der Studie (>Abb. 1):

1. Die Bestimmung von klinischen Blutparametern, z.B. Blutzucker und Cholesterin

2. Standardisierte Fragebogen zu Ernährungsverhalten und -motiven, Gesundheit, körperlicher Aktivität und gesundheitsbezogenen Einstellungen

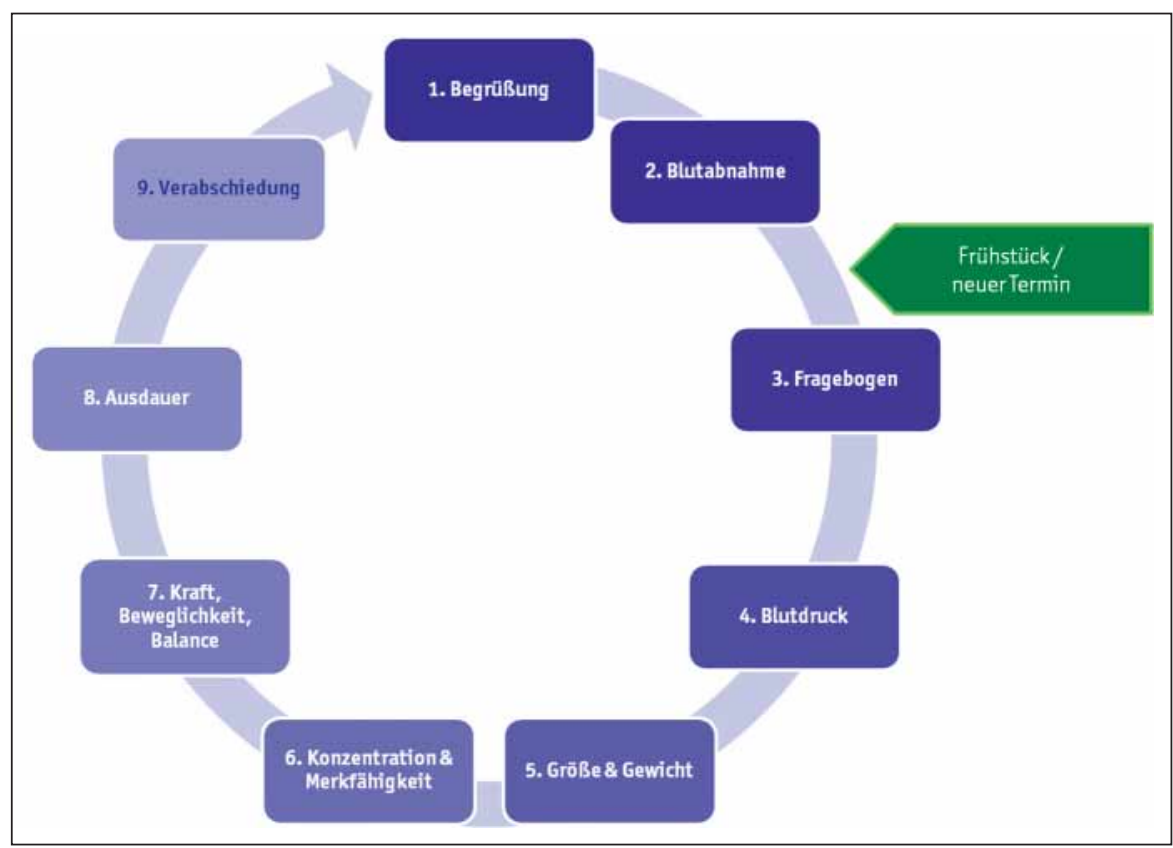

Abb.1 Ablauf der Konstanzer-Life-Studie zur Erforschung des normalen Essverhaltens. 
3. Anthropometrische Parameter: Größe, Gewicht, Taillen- zu Hüftumfang, Körperfettanteil

4. Untersuchungen zur Bestimmung der funktionellen Fitness mit einer Fahrradergometrie, Messungen von Kraft, Beweglichkeit und Gleichgewicht sowie von Konzentration und Gedächtnis

Insgesamt werden rund 1200 Erwachsene ohne schwerwiegende gesundheitliche Einschränkungen untersucht. Die Teilnahme ist freiwillig und alle TeilnehmerInnen er- halten einen Gesundheitspass mit individuellen Rückmeldungen auf Basis der persönlichen Ergebnisse.

Die Konstanzer Life-Studie ist ein Teil des Forschungsprojektes EATMOTIVE unter der Leitung von Prof. Dr. Renner und unter Mitwirkung von Prof. Dr. Schupp (Neurowissenschaften) und Prof. Dr. Bürkle (Molekulare Toxikologie) der Universität Konstanz. Projektmanagement: Dr. Verena Klusmann, Dr. Gudrun Sproesser, Dr. María Moreno-Villanueva, Dipl.-Ing. agr. Bettina Ott. Förderung durch: Bun- desministerium für Bildung und Forschung (BMBF), Universität Konstanz. Kooperationspartner: Stadt Konstanz, Deutsches Rotes Kreuz Landkreis Konstanz. Schirmherren: Rektor der Universität Konstanz Prof. Dr. Ulrich Rüdiger, Oberbürgermeister der Stadt Konstanz Horst Frank.

Korrespondenzadresse

Prof. Dr. Britta Renner

Arbeitsgruppe Psychologische Diagnostik und Gesund-

heitspsychologie, Universität Konstanz

78457 Konstanz

E-Mail: britta.renner@uni-konstanz.de

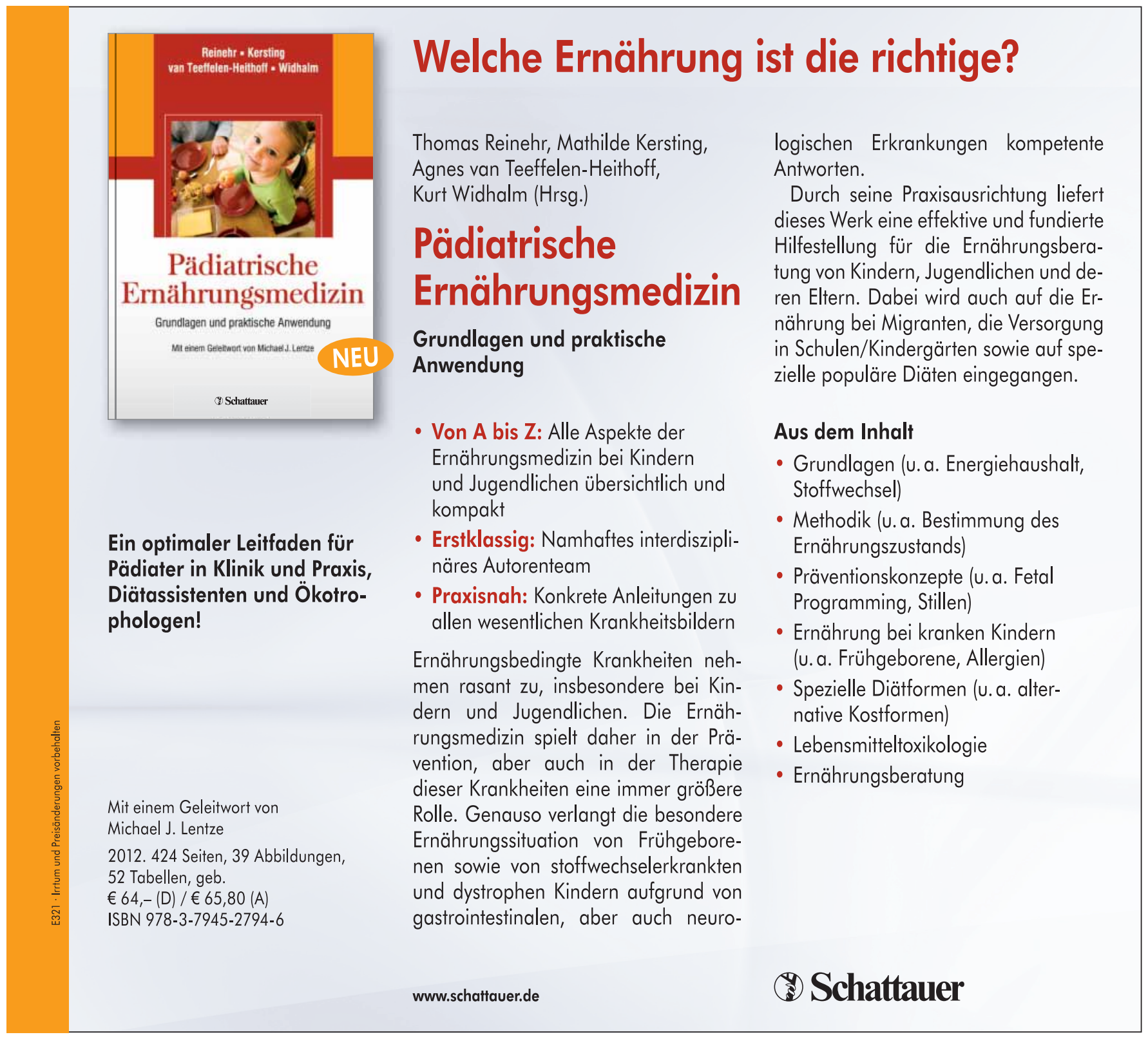

